\title{
Enabling Sleep Mode in Backbone IP-Networks: a Criticality-Driven Tradeoff
}

\author{
Aruna Prem Bianzino ${ }^{a, b}$, Claude Chaudet $^{a}$, Stefano Moretti ${ }^{c}$, Jean-Louis Rougier ${ }^{a}$, \\ Luca Chiaraviglio $^{d}$, Esther Le Rouzic ${ }^{e}$ \\ a) Institut Télécom, Télécom ParisTech, CNRS LTCI UMR 5141, Paris, France \\ b) Consorzio Nazionale Interuniversitario per le Telecomunicazioni, Torino, Italy \\ c) CNRS UMR 7243, Université Paris-Dauphine, Paris, France \\ d) Electronics Department, Politecnico di Torino, Italy \\ e) Orange Labs, Networks and Carriers, Lannion, France \\ Email: bianzino@telecom-paristech.fr
}

\begin{abstract}
The energy consumption of network devices, and, as a consequence, of communication networks, is generally independent from their level of utilization, which results in a waste of energy when the network is lightly loaded. Ideally the consumption of a network should be proportional to the amount of traffic it conveys. The most straightforward way to enforce such a proportionality between the network energy consumption and its utilization level, is to dynamically adapt the status of network devices to the load, forcing a subset of them to enter a sleep state during the low activity periods.

We present in this paper an algorithm to dynamically put links into a sleep state, based on a cooperative-game approach, named "L-Game". Our approach decides which links can be switched off based on a measure of the criticality of each link expressed as its Shapley value. This measure combines topological aspects and traffic conditions. Simulation results on real network scenarios show that our solution achieves a better trade off between energy saving and Traffic Engineering than other legacy approaches.
\end{abstract}

\section{INTRODUCTION}

Communication devices are generally not able to adapt automatically their energy consumption to the load they have to sustain. Thus, the energy consumed by communication networks is almost constant and independent from their level of utilization. "Resource consolidation" designates a set of techniques that carefully select a subset of network devices to route the actual traffic demand, while forcing the others to a sleep state. It allows the network to reach, on average, an energy consumption proportional to its utilization level [1]. However, resource consolidation must preserve Quality of Service (QoS): a minimum QoS level may be guaranteed, for instance, by imposing a maximum link utilization - a common practice in operator networks.

This resource consolidation paradigm is the basis of several works in the green communication field, many of which propose algorithms to put links into sleep mode. Previous articles aimed at solving the corresponding optimization problem [2], at designing distributed solutions, in which nodes take independent decisions [3], possibly sharing knowledge to improve the result [4].

We propose, in this paper, to select active and sleeping links based on their Shapley value [5], [6], a measure of their "criticality" when fulfilling the network transit tasks. The Shapley value takes into account both the network topology and the current traffic conditions. These values can then be used to identify the less critical links, which will be the first to be switched off. This method achieves an energy consumption that is almost proportional to the network load, almost reaching the proportionality objective, while preserving the global network performance objectives.

The idea of criticality-driven resource consolidation, considering the Shapley value as criticality for network devices, was introduced in [6], considering only nodes. This work extends the original idea considering links, and criticality of links in the network, which is more interesting for practical perspectives, as on/sleep state transitions are easier for links than for nodes, considering current and foreseen network devices.

Simulation results obtained on real network scenarios show that our solution is able to achieve a good trade off between energy consumption and Traffic Engineering (TE), reducing of about $50 \%$ the network energy consumption, while keeping link load under control.

\section{Shapley Value as Link CRiticality}

\section{A. Rationales}

In this work, we model the communication links of a backbone IP network as the set of players in a cooperative game. Partitions of these links, i.e., subsets of links that are active while the others are asleep, can be seen as coalitions. The worth of a coalition is defined as the amount of traffic that can be accommodated by the corresponding network configuration. Links can be ranked by the average worth they bring to the different network configuration by using a classical tool of cooperative game theory: the Shapley value.

As presented below, the Shapley value of a player $a$ is computed as the average worth brought by the addition of player $a$ over all possible sub-coalitions (i.e., all combination of subsets of all the players, except $a$ ). Removing a player from a coalition corresponds to removing a link from the network. This deletion may lead to the partition of the network (i.e., 
loss of connectivity between different parts of the network), which in turn limits the maximum flow that the network can convey. The "importance" of a link is thus computed based on the additional traffic that can be accommodated thanks to the addition of the link (or how much traffic is lost when deleting the link, which is equivalent).

The benefits of considering Shapley values as a measure of the importance of a link in the network are twofold. (i) First, with the definition we adopt, both traffic and topology aspects are considered. Some links can be quite "critical" for the connectivity of the network but some of these critical links might be more important than others considering the amount of traffic that they transport. As discussed in [6], previous works that computed network elements centrality/criticality were usually solely based on topological aspects. If a purely topological point of view makes sense for various applications (social networks etc.), we believe that it is not appropriate from a traffic engineering perspective. (ii) Then, the Shapley value is defined as an average value over all possible sub-coalitions. In other words, the algorithm does not consider a single best path (w.r.t. some static link weights) but many possible alternative paths (as explained in Sec. II-B). The alternate paths may be used to better balance the load in the network, for instance. All these properties indicate that the Shapley value seems perfectly suited to reflect the tradeoff between saving energy and preserving QoS.

\section{B. Computation of the Shapley Value}

Because of space constraints, we only provide basic material about the Shapley value and its computation and we refer the interesting reader to, e.g., [7].

The network studied is represented by a graph $G=\langle N, E\rangle$ where $N$ is the set of vertices (i.e., routers) and $E$ is the set of (undirected) links $e$ of the form $\{i, j\}$ with $i, j \in N, i \neq j$.

For any subset $S \subseteq E$, we can define a reduced graph $G_{S}=\left\langle N_{S}, S\right\rangle$ corresponding to the links of $S$ and where the vertices correspond to either the origin or the destinations of these links. The cardinality of a set $S$ is denoted by $|S|$.

A path is defined as a sequence of consecutive nodes $\left(i_{0}, i_{1}, \ldots, i_{k}\right)$, s.t. $\forall a,\left\{i_{a}, i_{a+1}\right\} \in E$. It is acyclic if no vertex appears twice: $\forall a, b \in\{0,1, \ldots, k\}, a \neq b \Rightarrow i_{a} \neq i_{b}$. We denote by $\mathcal{M}_{G}(\{i, j\})$ the set of all the acyclic paths between $i$ and $j$ in graph $G$.

Given a path $p=\left(i_{0}, i_{1}, \ldots, i_{k}\right)$ in $G$, we say that a link $e=\{i, j\}$ belongs to $p$ if and only if $\left\{i=i_{s}, i_{s+1}=j\right\}$ for some $s \in\{0, \ldots, k-1\}$ and we denote by $S(p)$ the set of links which belong to the path $p$, i.e., $S(p)=\{e \in E: e$ belongs to $p$ \}.

We consider that traffic matrices are available for the network represented by $G$. The elements of such a traffic matrix, $T=\left(t_{i, j}\right)_{i, j \in N}$ are quantifications of the volumes of traffic generated by endpoints connected through a source router $i$ and consumed by endpoints connected through a destination router $j$. The amount of traffic that a sub-graph $G_{S}$ (for any $S \subseteq E$ ) can accommodate, with respect to $T$, is defined by $\nu(S)=\sum_{i, j \in N_{S}} t_{i, j} \mathbf{1}_{G_{S}}(i, j)$, where $\mathbf{1}_{G_{S}}(i, j)=1$ whenever $i$ and $j$ are connected in the reduced graph $G_{S}$ (i.e., there exist a path in $G_{S}$ from $i$ to $j$ ) and zero otherwise. By convention $\nu(\emptyset)=0$.

As presented earlier, the game is built considering links as players (differently from [6], where the game was built considering nodes as players). There exist different equivalent formulations for the Shapley value [7]. As presented in [6], the formulation based on the so-called Harsanyi dividends (denoted $\lambda_{S}$ for any $S \subseteq E$ ) is interesting in term of computation. The Harsanyi dividends are computed recursively as $\lambda_{S}=\nu(S)-\sum_{K \subset S} \lambda_{K}$. They can be interpreted as the marginal surplus of a coalition (i.e., $S$ ) w.r.t. to the sum of the individual worths of smaller coalitions (i.e., $K \subset S$ ). The Shapley value of a link $e \in E$ is then defined as:

$$
\phi_{e}(\nu)=\sum_{S \subseteq E: e \in S} \frac{\lambda_{S}}{|S|}
$$

Let us consider two links $e_{1}$ and $e_{2}$ that are not adjacent, i.e., $e_{1} \cap e_{2}=\emptyset$. Then, it is clear that $\nu\left(\left\{e_{1}\right\} \cup\left\{e_{2}\right\}\right)=\nu\left(\left\{e_{1}\right\}\right)+$ $\nu\left(\left\{e_{2}\right\}\right)$ and thus $\lambda_{\left\{e_{1}\right\} \cup\left\{e_{2}\right\}}=0$. As a consequence, in order to compute the Shapley value of a link $e$, we don't need to consider all the possible coalitions $S \subset E$ but only the elements that represent valid paths (i.e., coalitions where adjacency brings "worth") in which the link participates.

Let us also denote by $\mathcal{P}_{k}\left(\mathcal{M}_{G}(\{i, j\})\right)$ the set composed by all the $k$-combinations of paths from the set $\mathcal{M}_{G}(\{i, j\})$, i.e., subsets of $\mathcal{M}_{G}(\{i, j\})$ composed of exactly $k$ elements. Following the approach introduced in [6], it can be proved that the Shapley value granted to a link $e \in E$ is $\phi_{e}(\nu)=$ $\sum_{i, j \in N} \phi_{e}(i, j)$, with

$$
\phi_{e}(i, j)=t_{i, j} \sum_{k=1}^{\left|\mathcal{M}_{G}(\{i, j\})\right|}\left(\sum_{P \in \mathcal{P}_{k}\left(\mathcal{M}_{G}\{i, j\}\right)} \frac{(-1)^{k+1}}{|\pi(P)|} \cdot \mathbf{1}_{e}(P)\right)
$$

where $\pi(P)=\bigcup_{p \in P} S(p)$ and $\mathbf{1}_{e}(P)=1$ if link $e \in \pi(P)$, and $\mathbf{1}_{e}(P)=0$ otherwise. Note that only acyclic paths are considered in the computation.

As an example of computation using relation (2), consider a graph with two paths between $i$ and $j$, namely $p=(i, A, j)$ and $q=(i, B, C, j)$. Moreover, suppose that $t_{i, j}=1$. Then, by relation (2) we have that the resulting Shapley values $\phi=$ $(\{i, B\},\{B, C\},\{C, j\},\{i, A\},\{A, j\})$ are:

$$
\begin{aligned}
\phi(i, j) & =\left(\left(\frac{1}{3}, \frac{1}{3}, \frac{1}{3}, 0,0\right)+\left(0,0,0, \frac{1}{2}, \frac{1}{2}\right)-\left(\frac{1}{5}, \frac{1}{5}, \frac{1}{5}, \frac{1}{5}, \frac{1}{5}\right)\right) \\
& =\left(\frac{4}{30}, \frac{4}{30}, \frac{4}{30}, \frac{9}{30}, \frac{9}{30}\right) .
\end{aligned}
$$

\section{CRiticality-Driven Resource Consolidation}

In this section, we define an algorithm to perform resource consolidation in backbone IP networks based on the criticality ranking defined by the Shapley value. In practice, the algorithm consists in progressively considering all the network links, one by one, for de-activation, following the Shapley ranking. It will be referred to as "L-Game" from now on.

When putting a link into sleep, we analyze the resulting network, all previously examined links remaining in the proper 
sleep/working state. If the resulting network remains able to accommodate the currently required traffic, with a maximum link load lower than a pre-defined threshold $(\theta)$, the link is placed into a sleep state, otherwise it is left in working state. The process then iterates until all the network links have been examined.

As briefly presented in the previous section, computing Shapley values can be achieved by considering all the paths to which a link participates as contributing coalitions, which requires theoretically to explore all the possible paths in the network. To reduce this computation complexity, we use two approximations:

- First, we limit the set of paths to explore using the techniques discussed in [6]. Indeed, the contribution of a path is inversely proportional to its length in Shapley value (as it will be seen in equation (1)). This means that we should be able to limit the maximum length of the considered paths without affecting too much the precision of the results. In practice, this limitation, which has been verified experimentally, makes sense as very long paths are unlikely in operational networks.

- Second, we also consider that routers are organized hierarchically (e.g., access/core) and that routing inside the network complies to the so-called valley-free property (using an analogy to BGP routing in the Internet [8]): i.e., traffic travels up the hierarchy once and then down the hierarchy once, but does not go back and forth between the hierarchical levels. This rule avoids traffic to transit via "access" routers. In the FT network depicted in Fig. 2, for instance, this rule will insure that access nodes $(8,9, \ldots$,

12) are not used to forward traffic between core routers 24 and 25.

As a summary, we first identify all the paths to explore, using a depth-first search procedure and imposing both the valley-free principle and a maximum length equal to the shortest path length plus a few hops. In the setups we explored, 2 additional hops give accurate results. We then compute the contributions of each path to the Shapley values based on the traffic matrix. We then sort the links by increasing Shapley value order and test each link de-activation iteratively.

\section{Performance Evaluation}

For performance evaluation, we compare the Shapley-based ranking with two other rankings introduced in [9]: (i) the Most Power (MP) ranking, which progressively considers links to be de-activated on the basis of decreasing link power consumption, and (ii) the Least Flow (LF) ranking, which follows instead an increasing order based on the traffic load to which links are subject, in the case in which all links are in working state ${ }^{1}$. To provide a relevant evaluation of the proposed algorithm, we tested it over two real network scenario, namely TIGER and FT.

\footnotetext{
${ }^{1}$ Note that in the case of the L-Game and LF rankings, the first link candidate to enter a sleep state is the one with lower ranking, while for the MP ranking the first link candidate to enter a sleep state is the one with higher ranking.
}

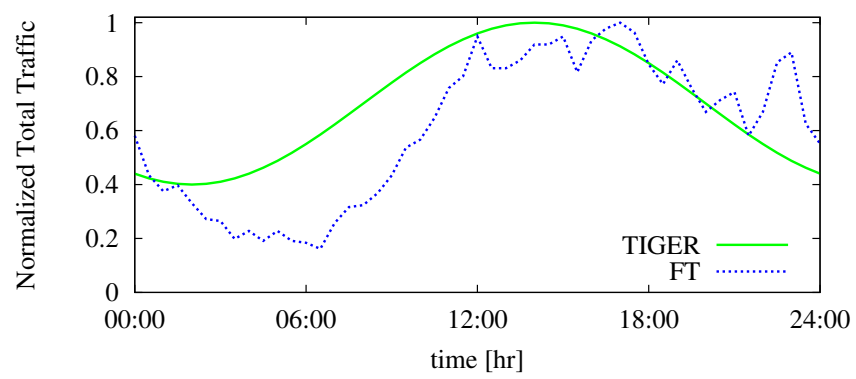

Fig. 1. Variation of the total traffic load versus time, normalized to the peak total traffic.

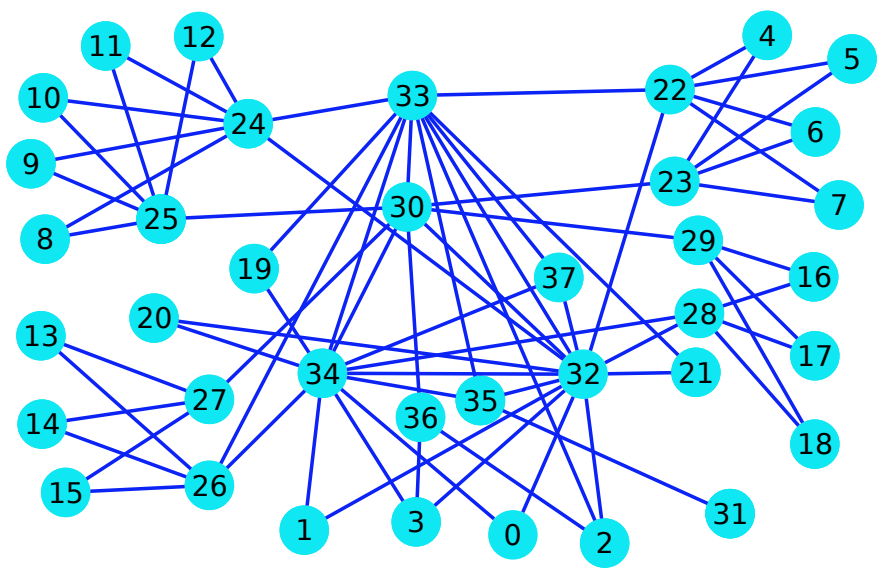

Fig. 2. Network topology of the FT scenario.

The TIGER scenario corresponds to an access/metro segment of a traditional ISP network. The network, whose topology is represented on Fig. 3, is organized in a hierarchical structure, including access nodes, which are source and destination of traffic requests, transit nodes, which route traffic for other nodes but not generate/receive traffic, and a traffic collection point, providing access to the ISP transport network and the Internet. For this scenario, a single traffic matrix was provided by the ISP, to which we applied a standard night/day trend [10]. The resulting normalized total traffic versus time in the network is reported in Fig. 1 by the green solid line. Fig. 3 represents the criticality granted by the three considered rankings to the network links (namely, L-Game, LF, and MP). Further details about the TIGER scenario are provided in [3], where it is referred to as "ISP 1".

The FT scenario represents an actual backbone IP network of France Telecom, whose topology is composed by 38 nodes and 72 bidirectional links, as reported in Fig. 2. For this scenario, the link capacities and lengths are provided. Finally, the operator disclosed the amount of traffic exchanged among each node pair as foreseen in year 2020 under a conservative assumption of user and traffic growth. The total, normalized variation of traffic is reported in Fig. 1 by the blue dotted line.

Tab. I resumes the main characteristics of the two reference scenarios. Traffic is routed on the minimum cost paths, 

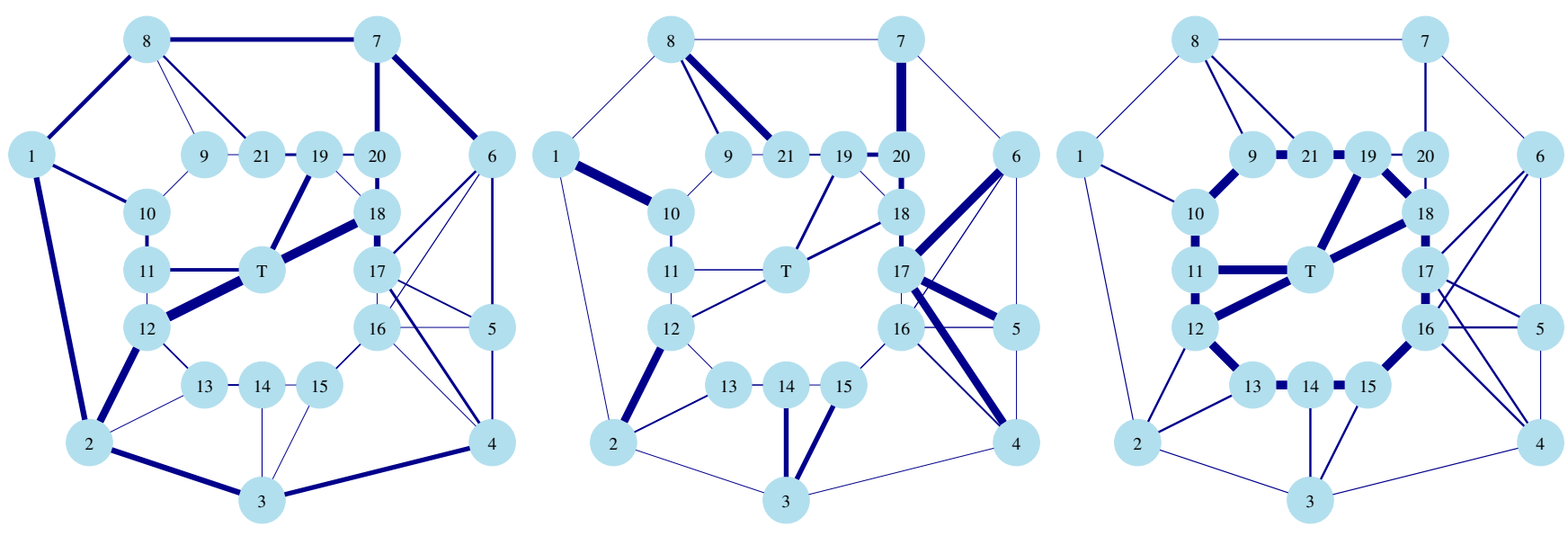

Fig. 3. Graphical view of the different rankings for the TIGER network scenario, for the peak hour traffic: the thicker a link, the higher its importance is with respect to the considered rank (from left to right: L-Game, LF, MP).

TABLE I

MAIN CHARACTERISTICS OF THE REFERENCE SCENARIOS.

\begin{tabular}{|l|c|c|c|}
\hline Parameter & Symbol & TIGER & FT \\
\hline Maximum Link Load [\%] & $\theta$ & 75 & 50 \\
\hline Number of nodes & $|N|$ & 22 & 38 \\
\hline Number of Links & $|L|$ & 40 & 72 \\
\hline Average link length [km] & $E\left[l_{i j}\right]$ & 43 & 278 \\
\hline
\end{tabular}

considering routing weights inversely proportional to the link capacities. The performance statistics (such as link loads etc.) are averages over all the Traffic Matrices (one every 30 minutes). Aggregated traffic flows are considered, and hence no fast traffic fluctuations are considered in these simulations.

\section{A. Energy Saving and QoS}

As performance metrics, we use the (i) energy saving on the one side, computed with respect to the configuration in which all links are powered on all time, and the (ii) average link load on the other side, as indicator of the QoS offered by the network. A maximum link load, denoted by $\theta$ hereafter, is imposed, as common practice in ISP networks, as minimum QoS guarantee.

In order to evaluate the network energy consumption, we use here the energy model of [11] as it is representative for actual current network devices. In this model, each IP link consumes an amount of power corresponding to a pair of transponders and a pair of IP interface ports. Each $10 \mathrm{Gbps}$ transponder consumes $37 \mathrm{~W}$ and each 1 Gbps port consumes $10 \mathrm{~W}$.

We simulated the effect of the resource consolidation algorithm presented above on both scenarios. The achievable energy saving when considering the three different rankings is reported in the upper part of Fig. 4 for the TIGER scenario, and in the upper part of Fig. 5 for the FT scenario. The corresponding average link loads are reported in the lower part of Fig. 4 for the TIGER scenario, and in the lower part of Fig. 5 for the FT scenario.

For both scenarios, two main time zones may be identified: a day time zone and night time zone. The day time zone is characterized by higher average link loads, representing the main limitation to the resource consolidation. In the night time zone, link loads are much lower, and the network connectivity represents the main limitation to resource consolidation. Of course, the possible energy savings that can be achieved are higher during the night time zone. This consideration is true for all the three considered rankings, the difference among which lies in the different trade off they are able to achieve between QoS and energy saving.

As already suggested in [9], MP is able to achieve an energy saving higher than LF, at the price of higher average link load, as it tries to put asleep the most power-hungry links (regardless of their criticality). The L-Game ranking is able to achieve an energy saving comparable to the one obtained by the MP ranking, but using a smaller set of configurations with respect to MP (i.e., 2 against 6, in the TIGER scenario, and 11 against 20 in the FT scenario), and requiring a smaller number of network reconfiguration (i.e., 2 against 12, in the TIGER scenario, and 24 against 32 in the FT scenario, per day). ${ }^{2}$ At the same time, the L-Game ranking guarantees the lower link load among the three considered rankings, and the better link load distribution (Fig. 6 reports the link load distribution for the FT scenario, for the peak traffic request. As the link load is limited by the threshold $\theta$, link load is normalized with respect to $\theta$. Similar results have been obtained for the TIGER scenario, omitted here for lack of space).

The L-Game ranking is able to achieve a better QoS performances (w.r.t. link loads) than the MP one, as MP drives the resource consolidation process keeping into account only the energy cost of links, but not the network topology, nor the traffic flowing on it. At the same time, the LF ranking requires more frequent network reconfigurations than MP and L-Game, as it is based on the link load, which frequently and strongly varies in time, and as it only takes into account the amount of carried traffic, in an agnostic configuration, while the L-Game

\footnotetext{
${ }^{2}$ Frequently turning on and off links may affect the network routing, since each reconfiguration requires a new convergence transient.
} 

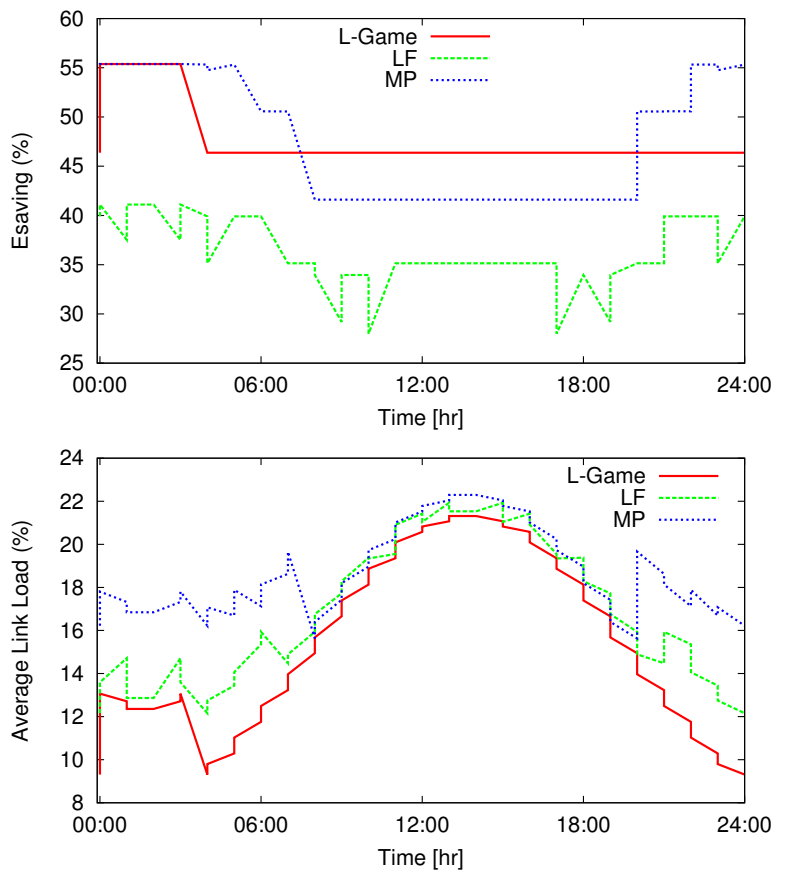

Fig. 4. Achievable energy saving (top), and corresponding average link load (bottom), for the TIGER network scenario, for different link rankings.
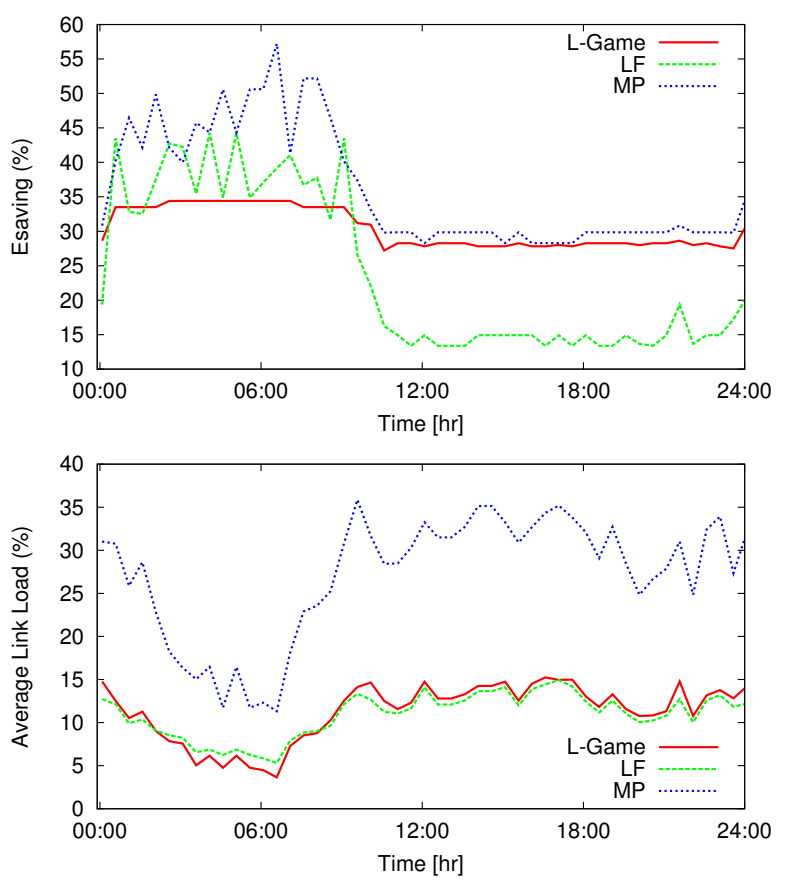

Fig. 5. Achievable energy saving (top), and corresponding average link load (bottom), for the FT network scenario, for different link rankings.

ranking accounts also for the position of link in the network, and for all possible network configurations.

\section{CONCLUSIONS}

We presented a novel approach to drive the resource consolidation process by the criticality of links in networks, namely

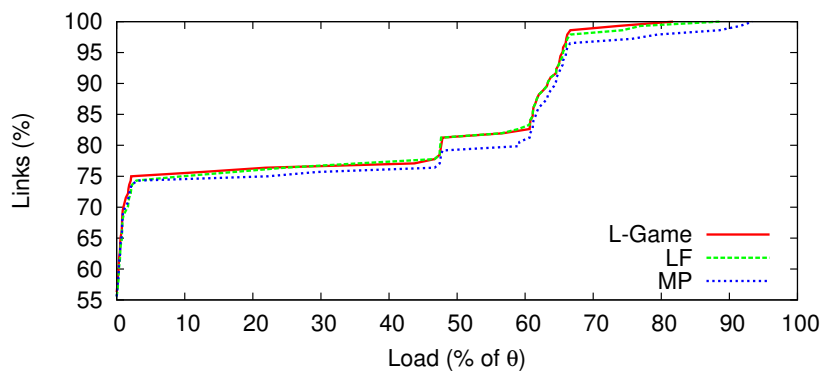

Fig. 6. Distribution of the link loads for the FT network scenario, for different link rankings, for the peak traffic request.

"L-Game". The adopted concept of criticality, based on a cooperative-game approach, is able to take into account at the same time both (i) the network topology, and (ii) the amount of traffic that the network is required to deliver, unlike previous approaches. Simulation results, on real network scenarios, show that the L-Game is able to achieve a better trade off between energy saving and QoS, with respect to classical solutions, being able to reduce the network energy consumption of about a half, and limiting, at the same time, the traffic load on links.

\section{ACKNOWLEDGMENT}

The work described in this paper was funded in part by FP7 ETICS, FP7 TREND (grant agreement n. 257740), and EuroNF.

\section{REFERENCES}

[1] A. Bianzino, C. Chaudet, D. Rossi, and J. Rougier, "A Survey of Green Networking Research," IEEE Communication Surveys and Tutorials, 2012.

[2] A. Bianzino, C. Chaudet, F. Larroca, D. Rossi, and J. Rougier, "EnergyAware Routing: a Reality Check," in 3rd International Workshop on Green Communications (GreenComm3), (Miami, USA), Dec. 2010

[3] A. Bianzino, L. Chiaraviglio, and M. Mellia, "GRiDA: a Green Distributed Algorithm for Backbone Networks," in 2011 IEEE Online Green Communications Conference (GREENCOM 2011), September 2011. http://www.telematica.polito.it/chiaraviglio/papers/GRiDA.pdf.

[4] A. Cianfrani, V. Eramo, M. Listanti, M. Marazza, and E. Vittorini, "An Energy Saving Routing Algorithm for a Green OSPF Protocol," in IEEE INFOCOM Workshops, 2010, (San Diego, USA), Mar. 2010

[5] L. Shapley, "A value for n-person games," in Contributions to the Theory of Games II (K. H. and T. A.W., eds.), pp. 307-317, Princeton University Press, 1953.

[6] A. P. Bianzino, C. Chaudet, D. Rossi, J.-L. Rougier, and S. Moretti, "The Green-Game: Striking a Balance between QoS and Energy Saving," in 23rd International Teletraffic Congress (ITC), (San Francisco, CA, USA), IEEE / ACM, Sept. 2011.

[7] S. Moretti and F. Patrone, "Transversality of the Shapley value," Top, vol. 16, no. 1, pp. 1-41, 2008

[8] N. Feamster, H. Balakrishnan, and J. Rexford, "Some foundational problems in interdomain routing," in Proceedings of Third Workshop on Hot Topics in Networks (HotNets-III), (San Diego, CA, USA), Citeseer, Nov. 2004.

[9] L. Chiaraviglio, M. Mellia, and F. Neri, "Minimizing ISP Network Energy Cost: Formulation and Solutions," IEEE/ACM Transactions on Networking, to appear, 2011. http://www.telematica.polito.it/chiaraviglio/papers/GreenTon.pdf.

[10] What Europeans do at Night, "http://asert.arbornetworks.com/2009/08/ what-europeans-do-at-night/," 2009.

[11] F. Idzikowski, "Power consumption of network elements in ip over wdm networks," TU Berlin, TKN Group, Tech. Rep. TKN-09-006, 2009. 